\title{
Minimal incision-assisted full-thickness sampling with over-the-scope clip targeting intestinal neuronal malformation
}

Intestinal neuronal malformation (INM) is a rare and refractory pediatric disease [1]. Its definitive diagnosis is generally confirmed by an invasive full-thickness biopsy [2]. This biopsy is required because the nerve plexus is located in the deep submucosal and muscle layers, resulting in poor diagnostic ability with endoscopic suction biopsies [3]. In our experience, even specimens obtained by endoscopic submucosal dissection fail to provide an accurate histological evaluation owing to the burn effects. As a result, full-thickness specimens must be obtained to make a diagnosis of INM.

A new type of over-the-scope clip (OTSC), called a full-thickness resection device (FTRD; Ovesco Endoscopy, Tübingen, Germany), provides a moderate rate $(75 \%)$ of histologically complete resection, so indicating a need to modify procedures $[4,5]$. In this animal study, we introduced a productive endoscopic full-thickness sampling method with the original OTSC system targeting INM.

A flexible gastrointestinal endoscope was used. First, a 10-mm mucosal pocket was created in the lower rectum using a needle knife (KD-650Q; Olympus, Tokyo, Japan) until the muscle layer was visible ( Fig. 1 a). Next, after the artificial pocket had been anchored into the application cap with a retraction device (Anchor; Ovesco Endoscopy) that captured the exposed muscle layer, the OTSC was successfully deployed ( $\triangleright$ Fig. 1 b). A $10-\mathrm{mm}$ incision was then made with the needle knife in the muscle layer immediately above the clip to prevent slippage of the snaring device. Finally, a full-thickness resection was completed with the snare, without complications, using the Endo Cut $\mathrm{Q}$ mode on an electric generator
(VIO300D; ERBE, Tübingen, Germany) ( $\triangleright$ Fig. $1 \mathrm{c}, \mathrm{d}$; $>$ Video 1 ).

A $10-\mathrm{mm}$ specimen with a sufficient muscle layer was acquired ( $>$ Fig. 2). Histological examination revealed an adequate full-thickness layer including the myenteric plexus and ganglia cells (> Fig.3). This study emphasizes that a minimal incision-assisted OTSC procedure can facilitate full-thickness sampling and minimally invasive diagnosis of INM.

Endoscopy_UCTN_Code_TTT_1AO_2AC

Competing interests

None
The Authors

Noriko Nishiyama ${ }^{1}$, Hirohito Mori' Hideki Kobara ${ }^{1}$, Shintaro Fujihara', Maki Ayaki ${ }^{1}$, Yumi Miyai ${ }^{2}$, Tsutomu Masaki ${ }^{1}$

1 Department of Gastroenterology and Neurology, Faculty of Medicine, Kagawa University, Kagawa, Japan

2 Department of Diagnostic Pathology, Faculty of Medicine, Kagawa University, Kagawa, Japan

\section{Corresponding author}

\section{Noriko Nishiyama, MD, PhD}

Department of Gastroenterology and Neurology, Faculty of Medicine, Kagawa University, 1750-1 Ikenobe, Miki, Kita, Kagawa 761-0793, Japan

Fax: +81-87-8912158

n.nori.ocean@gmail.com

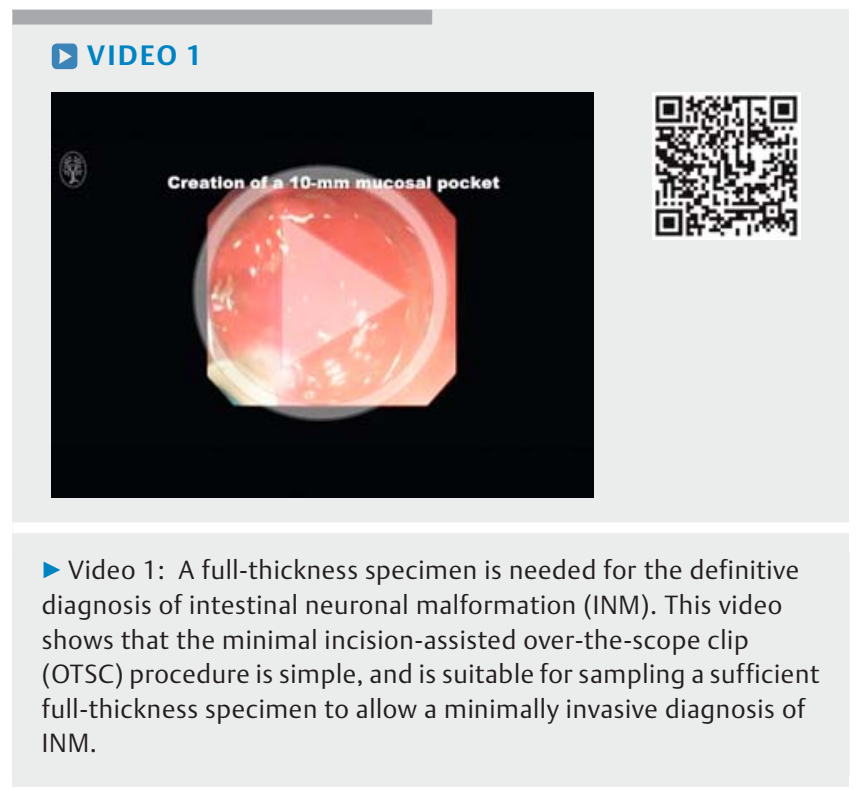



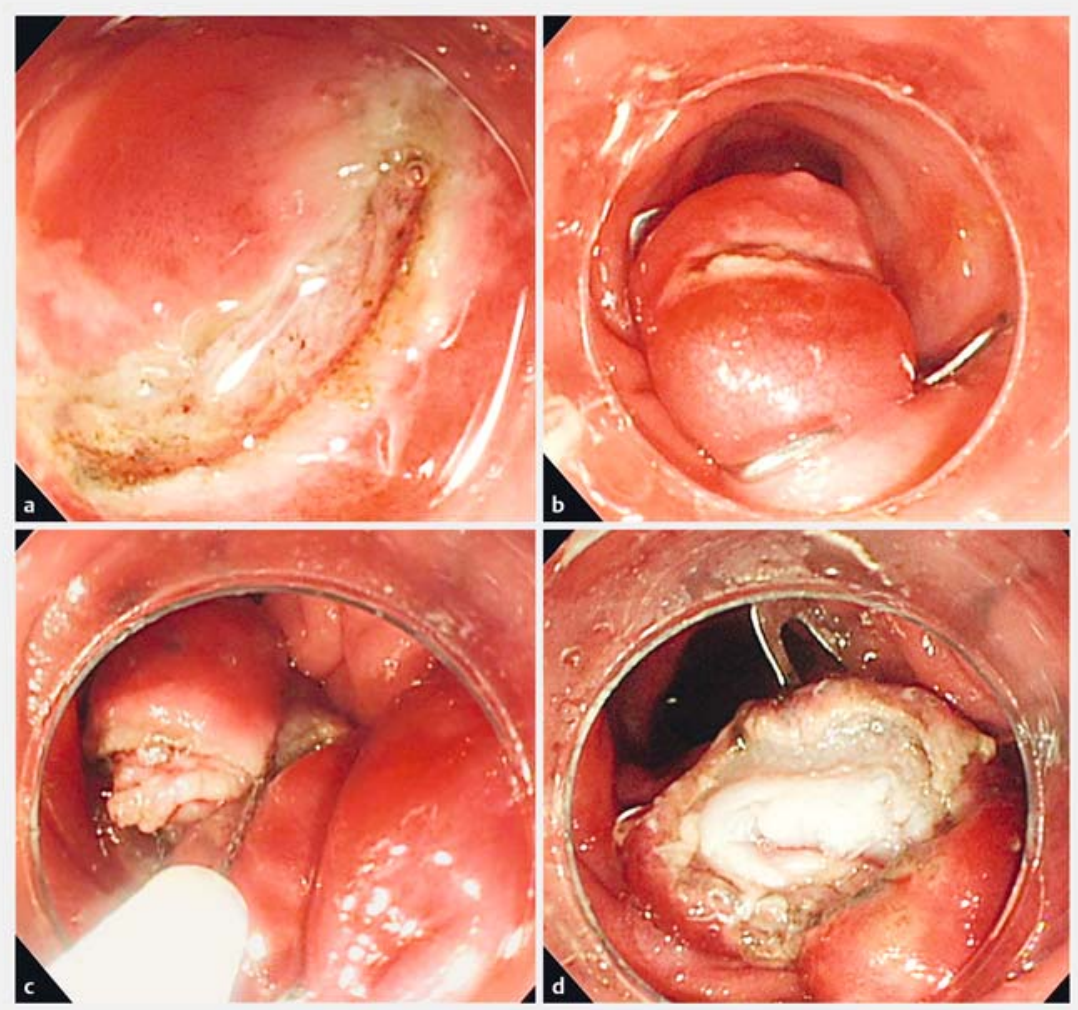

- Fig. 1 Endoscopic images showing: a a 10-mm pocket with the muscle layer exposed that was created with a needle knife; b successful over-the-scope clip (OTSC) deployment with an anchor assist that captured the exposed muscle layer of the pocket; $\mathbf{c}$ complete full-thickness resection using a snare after a $10-\mathrm{mm}$ incision had been made in the muscle layer immediately above the clip to prevent slippage of the snare; $\mathbf{d}$ a full-thickness defect that was closed by deployment of the OTSC.

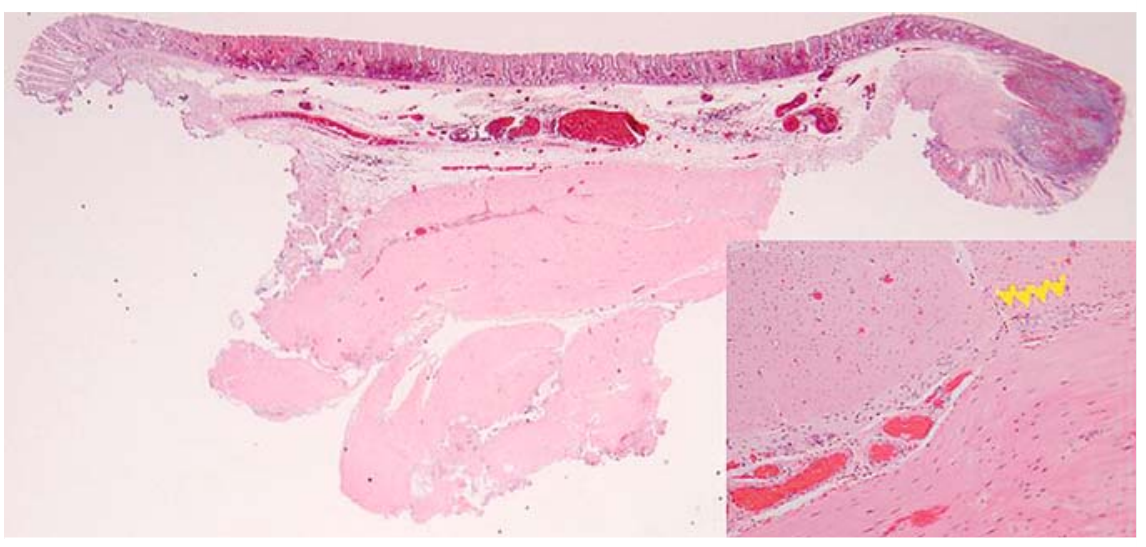

- Fig. 3 Histology of the specimen stained with hematoxylin and eosin (H\&E) showing the full-thickness layers with the presence of the internal circular and external longitudinal muscle layers, and the neurogenic plexus containing ganglia cells (yellow arrows).

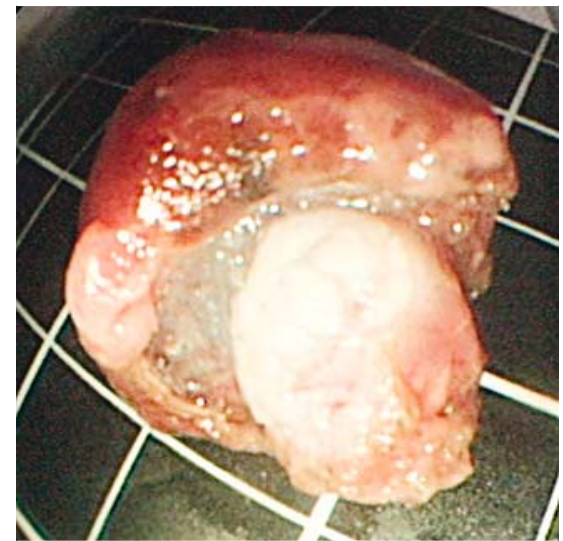

- Fig. 2 Macroscopic view showing the full-thickness resected specimen with a sufficient amount of muscle layer.

\section{References}

[1] Qadir I, Salick MM, Barakzai A et al. Isolated adult hypoganglionosis presenting as sigmoid volvulus: a case report. J Med Case Rep 2011; 5: 1

[2] Kobayashi H, Yamataka A, Lane G] et al. Pathophysiology of hypoganglionosis. Pediatr Gastroenterol Nutr 2002; 34: 231 232

[3] Yunis EJ, Dibbins AW, Sherman FE. Rectal suction biopsy in the diagnosis of Hirschsprung disease in infants. Arch Pathol Lab Med 1976; 100: 329-333

[4] Snauwaert C, Jouret-Mourin A, Piessevaux H. Endoscopic full-thickness resection of a nonlifting adenoma in an ileal pouch using an over-the-scope full-thickness resection device. Endoscopy 2015; 47: E344 - E345

[5] Schmidt A, Bauerfeind P, Gubler C et al. Endoscopic full-thickness resection in the colorectum with a novel over-the-scope device: first experience. Endoscopy 2015; 47: 719 725

\section{Bibliography}

DOI http://dx.doi.org/10.1055/s-0043-100626

Endoscopy 2017; 49: E103-E104

(C) Georg Thieme Verlag KG

Stuttgart · New York

ISSN 0013-726X 